\title{
Computational support on the development of nuclear heating calorimeter detector design
}

\author{
$1^{\text {st }}$ Klemen Ambrožič \\ Reactor physics division \\ Jožef Stefan Institute \\ Ljubljana, Slovenia \\ klemen.ambrozic@ijs.si \\ $5^{\text {th }}$ Luka Snoj \\ Reactor physics division \\ Jožef Stefan Institute \\ Ljubljana, Slovenia \\ luka.snoj@ijs.si
}

\author{
$2^{\text {nd }}$ Damien Fourmentel \\ DEN/DER/SPESI/LDCI \\ CEA Cadarache \\ Saint-Paul-lez-Durance, France \\ damien.fourmentel@cea.fr
}

\author{
$3^{\text {rd }}$ Hubert Carcreff \\ DEN/DANS/DM2S/SERMA \\ CEA Saclay \\ Gif sur Yvette, France \\ hubert.carcreff@cea.fr
}

\author{
$4^{\text {th }}$ Vladimir Radulović \\ Reactor physics division \\ Jožef Stefan Institute \\ Ljubljana, Slovenia \\ vladimir.radulovic@ijs.si
}

\begin{abstract}
Heating due to energy deposition of intense ionizing radiation in samples and structural materials of nuclear reactors poses severe limitations in terms of cooling requirements for safe reactor operation, especially in high neutron and gamma flux environments of material testing fission reactors (MTRs) and novel fusion devices. A bilateral CEA-JSI research project was launched in 2018 with the objective to measure the gamma heating rates in standard reactor-related materials (graphite, aluminium, stainless steel and tungsten) as well as fusionrelevant materials (low-activation steel Eurofer-97 and Nb3Sn superconductor) in the JSI TRIGA reactor my means of gamma calorimeters. The calorimeter design will be based on the the CALMOS-2 calorimeter developed at the CEA and used to perform gamma heating measurements in the OSIRIS MTR in Saclay.

In order to optimize the detector response inside the JSI TRIGA reactor field and not to perturb the measurement field, a detailed computational analysis was performed in terms of energy deposition assessment and measurement field perturbation using the MCNP v6.1 code, and in terms of heat transfer using the COMSOL Multiphysics code.

The abovementioned activities enabled us to finalize the detector design with the experimental campaign planned for the end of year 2019.
\end{abstract}

Index Terms-Nuclear heating, calorimetry, Nb3Sn, Eurofer97, TRIGA

\section{INTRODUCTION}

During operation of fission and fusion reactors, high levels of ionizing radiation are produced. In case of charged particle radiation, such as fission products and electrons, most of the energy is deposited locally. Neutral particles such as neutrons and gamma rays can on the other hand travel a significant distance before interaction with matter. The energy deposition mechanisms for neutrons and gamma rays also differ, with neutrons predominantly depositing energy by scattering on nuclei with low mass number, while gamma rays mainly interact with shell electrons, the reaction cross section increasing with

The paper is prepared in a scope of a billateral project "Analysis of nuclear heating in a reactor" funded by the Slovenian Research Agency and CEA. the atomic number [1]. In case of thermal fission reactors it is desirable for the neutrons to thermalize in the coolant, which is therefore comprised of low atomic mass materials. This in turn means that the gamma field is not significantly attenuated by the reactor coolant, but rather in reactor structural and sample materials, which leads to their heating and temperature increase. In high flux reactors the heating levels can reach levels exceeding $10 \mathrm{~W} \mathrm{~g}^{-1}$, leading to significant temperature increases and additional cooling requirements. In future fusion reactors, superconducting materials will be used which must be maintained at cryogenic temperatures. Additional gamma heating will represnent an important limitation for fusion devices, as the required plant cooling power is around 100 times larger than the actual cooling power. Knowledge of these heating values for neutron and gamma radiation will assist in the mitigation of the abovementioned technical difficulties at the planning stages of a specific experiment or in novel reactor designs.

Experimental determination of heating values in said materials with sufficient accuracy requires the experiments to be performed in a well characterized radiation field. The neutron and gamma fields inside the irradiation positions of the Jožef Stefan Institute (JSI) TRIGA Mark II reactor have been well characterized, both experimentally by means neutron activation dosimetry and miniature fission chamber techniques [2]-[4] for neutrons as well as TLD and ionization chamber measurements for gamma rays [5], [6].

The measurements will be performed using calorimetric methods, based on the CALMOS-2 measurement principles [7], [8]. The calorimeters will consist of a pedestal, containing the sample material and a heat sink. A temperature difference $\Delta T$ is measured between the pedestal with sample material (hot side) and heat sink at the detector bottom (cold side). A reference temperature difference $\Delta T_{R E F}$ with a detector without any sample serves as a measure of influence of the detector structures themselves. $\Delta \Delta T=\Delta T-\Delta T_{R E F}$ is used as a measure of deposited energy inside the material sample 
(Fig. 1).

The original CALMOS-2 sensors had both pedestals inte-

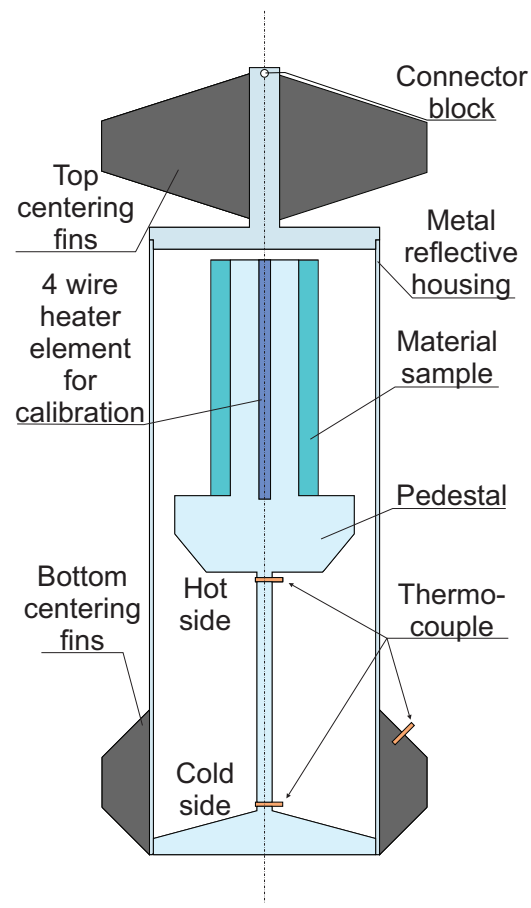

Fig. 1. Nuclear heating calorimeter sensor schematic for use inside the JSI TRIGA reactor.

grated into one sensor and were designed for specific use in the OSIRIS MTR reactor (full power $70 \mathrm{MW}$ ), where the neutron and gamma flux levels are an order of magnitude greater than those inside the JSI TRIGA reactor at full power $(250 \mathrm{~kW}$ in central irradiation channel). Due to different reactor geometry, an order of magnitude lower particle fluxes and natural convention in the JSI TRIGA reactor core, a completely new sensor based on the CALMOS-2 $\Delta \Delta T$ measurement principle is being designed. Several key aspects of the sensor had to be resolved before the final sensor design:

- The measurement sample should not perturb the radiation field by more than $5 \%$ and thus the determination of heating levels for all samples.

- Sensor should be compact enough to enable the performance of axial profile measurements along the JSI TRIGA fuel meat and to fit inside a wet irradiation channel with inner diameter of $34.5 \mathrm{~mm}$.

In the following sections the abovementioned tasks and their resolution using computational codes for particle transport and heat transfer calculations are described, leading to a finalized design of the calorimetric sensor.

\section{SAMPLE SIZE, HEATING LEVEL AND FIELD PERTURBATION}

The sample heating levels are determined by the choice of materials and reactor power levels. In order to obtain these values, a full JSI TRIGA reactor model was simulated using the MCNP v6.1 Monte Carlo particle transport code [9] and
ENDF/B VII.1 nuclear data libraries [10] without explicitly modeling the calorimeter detector or samples themselves, but rather calculating the heating rates along a cylindrical mesh inside the central irradiation channel (using the meshtally functionality) to determine the order of magnitude of the specific heating power for each sample material, as displayed in Fig. 2.

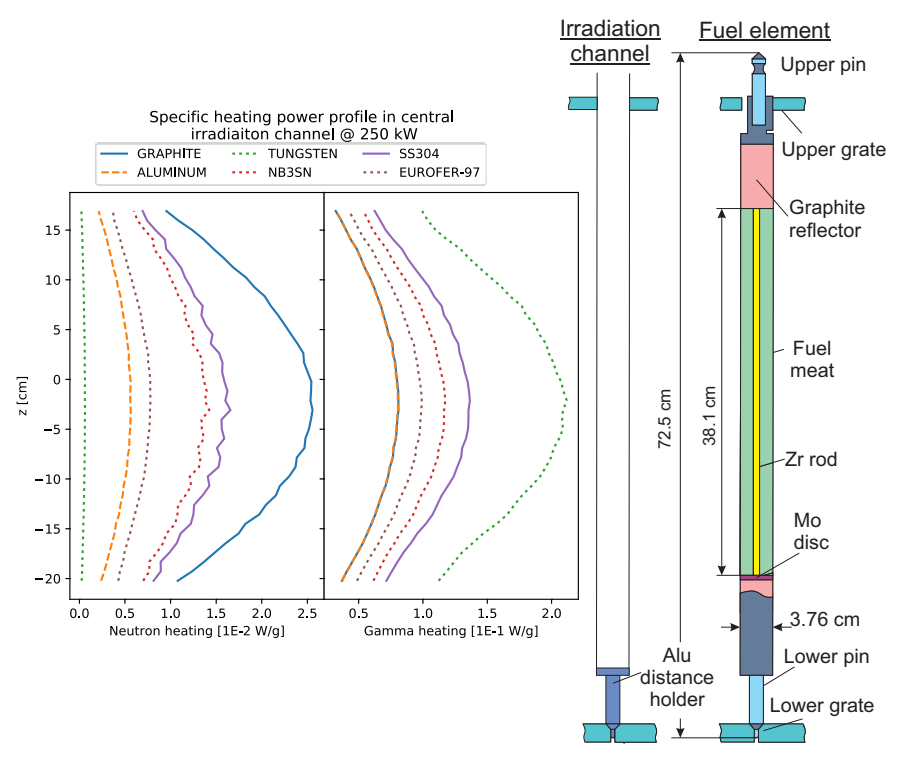

Fig. 2. Neutron and gamma axial heating profile in central channel of JSI TRIGA reactor at full reactor power $(250 \mathrm{~kW})$, corresponding to fuel element meat and irradiation channel.

From the calculated results we observe that the gamma heating rates are an order of magnitude larger than the neutron heating rates, gamma rays being attenuated to a larger extent in the material and depositing more energy. This was therefore the limiting factor in terms of the perturbation of the radiation field by the material samples.

The material sample geometry is adopted from the CALMOS2 calorimeter, i.e. a short length of tube with an inner diameter corresponding to the pedestal for effective thermal contact (inner diameter of $3.2 \mathrm{~mm}$ ). The outer diameter is limited by the perturbation of the radiation conditions, i.e. the degree to which the sample itself affects the radiation field. A limiting value of $5 \%$ was chosen. The maximum permissible outer diameter was determined by a parametric analysis using MCNP v6.1. An isotropic gamma source with spectrum corresponding to that of the JSI TRIGA reactor central channel was modeled on a cylindrical surface around the sample, $37.54 \mathrm{~mm}$ in diameter (corresponding to the internal diameter of the irradiation channel, Fig. 2). Gamma flux surface tallies (MCNP type 1 tallies) were defined on the inner and outer cylindrical surface of the modeled sample, with the gamma flux decrease calculated as the quotient between the two. The results of the parametric analysis are displayed in Fig. 3.

A single pedestal design will be used for all the sample materials. This means that the sample heating power levels should be of the same order of magnitude in order for the 


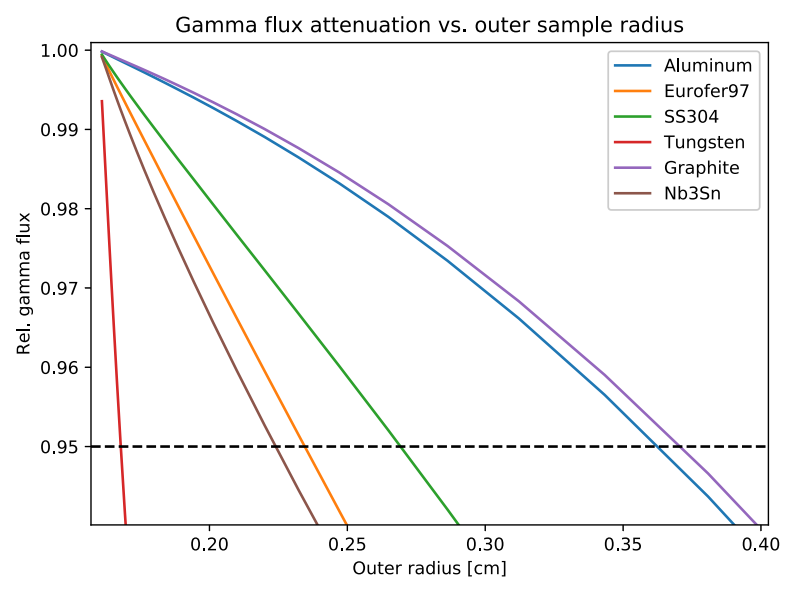

Fig. 3. Gamma flux attenuation versus sample material outer radius, with dotted line marking the $5 \%$ attenuation limit.

calorimeter to have similar response time constants. Therefore, the specific energy deposition averaged over the sample material as a function of the outer sample radius (MCNP type 6 tally) was calculated and its dependency displayed in Fig. 4.

Finally, a decision was made to set the heating value to

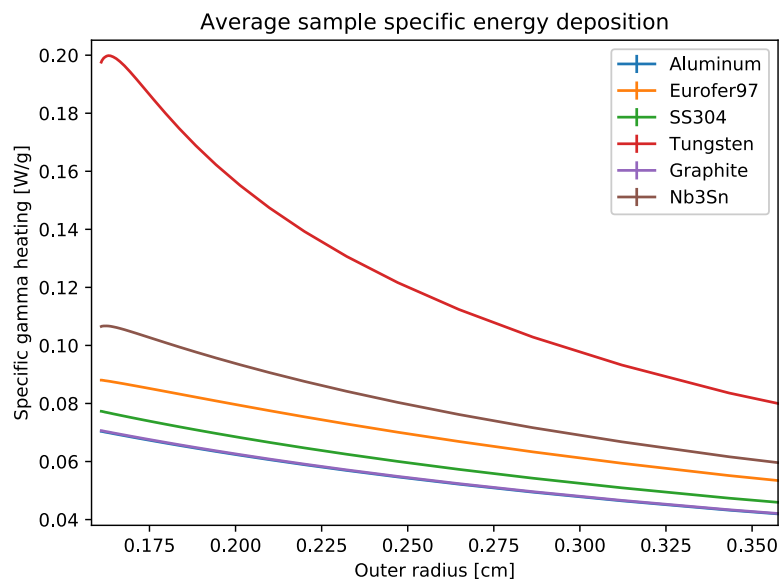

Fig. 4. Specific heating power, averaged over material sample versus outer sample radius.

roughly $0.04 \mathrm{~W} \mathrm{~g}^{-1}$ in central irradiation channel at the reactor power level of $100 \mathrm{~kW}$ setting the sample thicknesses accordingly (Table I). This then enabled us to perform heat transfer calculations for calorimeter design optimizations to estimate expected $\Delta \Delta T$ values.

TABLE I

SELECTED SAMPLE MATERIAL THICKNESSES.

\begin{tabular}{|l|l|l|l|l|l|l|}
\hline & $\mathrm{Al} 6063$ & Graphite & AISI 304L & Eurofer97 & Tungsten & $\mathrm{Nb} 3 \mathrm{Sn}$ \\
\hline Thickness [mm] & 2 & 2 & 1.1 & 0.76 & 0.1 & 0.6 \\
\hline
\end{tabular}

\section{HEAT TRANSFER SIMULATIONS}

The detector design itself is similar to the CALMOS-2 and CARMEN-1 [11] detectors designed for $2 \mathrm{Wg} \mathrm{g}^{-1}$ measurements in the OSIRIS reactor. Since two orders of magnitude lower heating values are expected, the calorimeter filling gas was switched from argon to xenon, which has by a factor of 3 lower thermal conductivity. Also the pedestal material was switched from aluminum to stainless steel, having a lower thermal conductivity, therefore increasing the temperature difference between the two pedestal thermocouples.

The heat transfer calculations were performed using the COMSOL multiphysics code (Fig. 5), in order to calculate the calorimeter heat exchange coefficient to the reactor pool water at a fixed temperature of $25^{\circ} \mathrm{C}$.

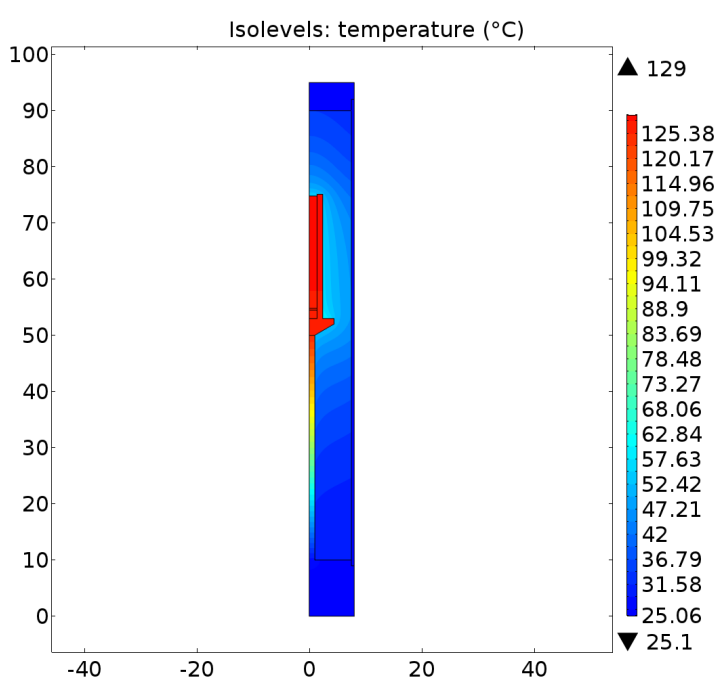

Fig. 5. 2D heat transfer simulation of the reference calorimeter detector (no sample).

The COMSOL code was also used do determine $\Delta \Delta T$ values for the lower and highest atomic number samples: graphite and tungsten, as summarized in Table II. The cal-

TABLE II

CALCULATED TEMPERATURE DIFFERENCES FOR SAMPLE MATERIALS WITH LOWEST AND HIGHEST ATOMIC NUMBER.

\begin{tabular}{|r|l|l|l|}
\hline & Reference & Graphite & Tungsten \\
\hline$\Delta T[\mathrm{~K}]$ & 100.7 & 135 & 112.8 \\
$\Delta \Delta T[\mathrm{~K}]$ & & 34.3 & 12.1 \\
\hline
\end{tabular}

culated temperature differences $\Delta \Delta T$ are sufficient, to be measured by the thermocouples, and the $\Delta T$ are low enough, so the the thermal radiation contribution can be neglected. The thermocouple locations were also evaluated, in order to place them in a low temperature gradient environment. Best locations were estimated by performing detailed 3D analysis of the heat transfer (Fig. 6) 


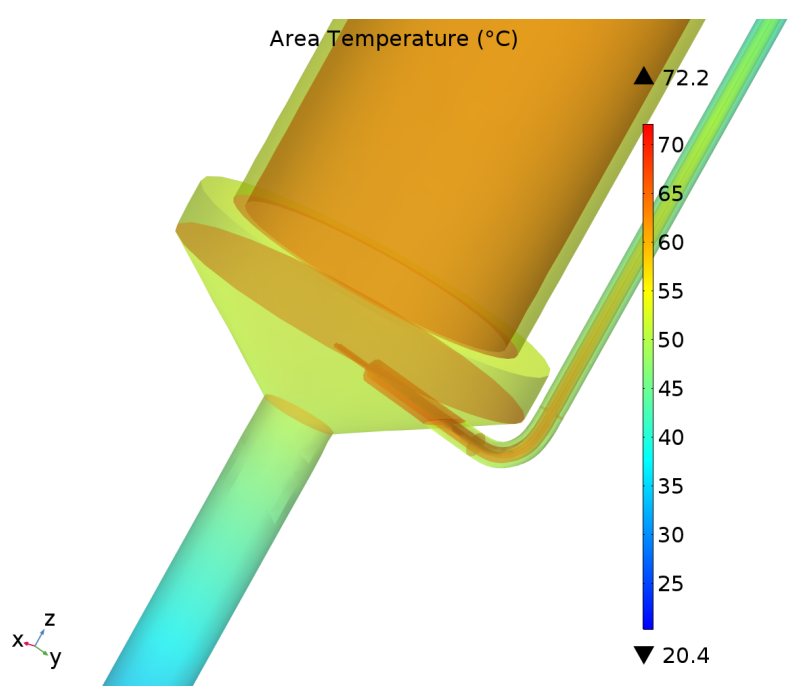

Fig. 6. Detailed 3D heat transfer analysis to determine optimum thermocouple positions on the pedestal.

\section{CONCLUSIONS}

A rigorous computational analysis of the radiation transport and heat transfer has been performed in order to optimize the nuclear heating calorimeter sensor for use inside the JSI TRIGA reactor central channel irradiation facility. In terms of heating values, a suitable energy deposition estimate had to be chosen in order to accommodate the diverse array of materials with a single pedestal design. The calorimeter design was then finalized by the aid of heat transfer calculations in order to have reasonable $\Delta \Delta T$ values.

\section{REFERENCES}

[1] F. Attix, Introduction to Radiological Physics and Radiation Dosimetry, ser. A Wiley-Interscience publication. Wiley, 1986. [Online]. Available: https://books.google.si/books?id=PL8971RdEfoC

[2] P. Filliatre, C. Jammes, L. Barbot, D. Fourmentel, B. Geslot I. Lengar, A. Jazbec, L. Snoj, and G. Žerovnik, "Experimental assessment of the kinetic parameters of the jsi triga reactor," Annals of Nuclear Energy, vol. 83, pp. 236 - 245, 2015. [Online]. Available: http://www.sciencedirect.com/science/article/pii/S0306454915002212

[3] T. Kaiba, V. Radulović, G. Žerovnik, L. Snoj, D. Fourmentel, L. Barbot, and C. A. a. Destouches, "Calculations to Support On-line Neutron Spectrum Adjustment by Measurements with Miniature Fission Chambers in the JSI TRIGA Reactor," in European Physical Journal Web of Conferences, ser. European Physical Journal Web of Conferences, vol. 170, Jan. 2018, p. 04012.

[4] Stancar, Žiga, Kaiba, Tanja, Snoj, Luka, Barbot, Loïc, Destouches, Christophe, Fourmentel, Damien, and Villard, Jean-François, "Reaction rate benchmark experiments with miniature fission chambers at the slovenian triga mark ii reactor," EPJ Web Conf., vol. 170, p. 04023, 2018. [Online]. Available: https://doi.org/10.1051/epjconf/201817004023

[5] K. Ambrožič, V. Radulović, L. Snoj, A. Gruel, M. L. Guillou, P. Blaise, C. Destouches, and L. Barbot, "Characterization of gamma field in the JSI TRIGA reactor," in European Physical Journal Web of Conferences, ser. European Physical Journal Web of Conferences, vol. 170, Jan. 2018, p. 04001.

[6] K. Ambrožič, A. Gruel, V. Radulović, M. L. Guillou, P. Blaise, C. Destouches, and L. Snoj, "Delayed gamma determination at the jsi triga reactor by synchronous measurements with fission and ionization chambers," Nuclear Instruments and Methods in Physics Research Section A: Accelerators, Spectrometers, Detectors and Associated Equipment, vol. 911, pp. 94 - 103, 2018. [Online]. Available: http://www.sciencedirect.com/science/article/pii/S0168900218312567
[7] Carcreff, H., Salmon, L., Lepeltier, V., Guyot, J.M., and Bouard, E., "Last improvements of the calmos calorimeter dedicated to thermal neutron flux and nuclear heating measurements inside the osiris reactor," EPJ Web Conf., vol. 170, p. 04002, 2018. [Online]. Available: https://doi.org/10.1051/epjconf/201817004002

[8] H. Carcreff, L. Salmon, J. Bubendorff, and V. Lepeltier, "First in-core simultaneous measurements of nuclear heating and thermal neutron flux obtained with the innovative mobile calorimeter calmos inside the osiris reactor," IEEE Transactions on Nuclear Science, vol. 63, no. 5, pp. 2662-2670, Oct 2016.

[9] J. T. Goorley, M. R. James, T. E. Booth, F. B. Brown, J. S. Bull, L. J. Cox, J. W. J. Durkee, J. S. Elson, M. L. Fensin, R. A. I Forster, and et al., Initial MCNP6 Release Overview - MCNP6 version 1.0. Los Alamos National Laboratory (LANL), Jun 2013 Report number: LANL Report LA-UR-13-22934. [Online]. Available: http://www.osti.gov/scitech/servlets/purl/1086758

[10] M. Chadwick, M. Herman, P. Obložinský, M. Dunn, Y. Danon, A. Kahler, D. Smith, B. Pritychenko, G. Arbanas, R. Arcilla, R. Brewer, D. Brown, R. Capote, A. Carlson, Y. Cho, H. Derrien, K. Guber, G. Hale, S. Hoblit, S. Holloway, T. Johnson, T. Kawano, B. Kiedrowski, H. Kim, S. Kunieda, N. Larson, L. Leal, J. Lestone, R. Little, E. McCutchan, R. MacFarlane, M. MacInnes, C. Mattoon, R. McKnight, S. Mughabghab, G. Nobre, G. Palmiotti, A. Palumbo, M. Pigni, V. Pronyaev, R. Sayer, A. Sonzogni, N. Summers, P. Talou, I. Thompson, A. Trkov, R. Vogt, S. van der Marck, A. Wallner, M. White, D. Wiarda, and P. Young, "Endf/b-vii.1 nuclear data for science and technology: Cross sections, covariances, fission product yields and decay data," Nuclear Data Sheets, vol. 112, no. 12, pp. 2887 2996, 2011, special Issue on ENDF/B-VII.1 Library. [Online]. Available: http://www.sciencedirect.com/science/article/pii/S009037521100113X

[11] D. Fourmentel, C. Reynard-Carette, A. Lyoussi, J. F. Villard, J. Y. Malo, M. Carette, J. Brun, P. Guimbal, and Y. Zerega, "Nuclear heating measurements in material testing reactor: A comparison between a differential calorimeter and a gamma thermometer," IEEE Transactions on Nuclear Science, vol. 60, no. 1, pp. 328-335, Feb 2013. 